Tuning procedure for traveling wave structures and its application to the C-Band cavities for SPARC photo injector energy upgrade

This content has been downloaded from IOPscience. Please scroll down to see the full text. 2013 JINST 8 P10010

(http://iopscience.iop.org/1748-0221/8/10/P10010)

View the table of contents for this issue, or go to the journal homepage for more

Download details:

IP Address: 155.97.178.73

This content was downloaded on 29/09/2014 at 12:47

Please note that terms and conditions apply. 


\title{
Tuning procedure for traveling wave structures and its application to the C-Band cavities for SPARC photo injector energy upgrade
}

\section{Alesini, ${ }^{a}, 1$ A. Citterio, ${ }^{c}$ G. Campogiani, ${ }^{b}$ L. Ficcadenti, ${ }^{b}$ M. Migliorati, ${ }^{b}$}

A. Mostacci, ${ }^{b}$ L. Palumbo, ${ }^{b}$ S. Persichelli ${ }^{b}$ and R. Zennaro ${ }^{c}$

\author{
a INFN, Laboratori Nazionali di Frascati, \\ P.O. Box 13, I-00044 Frascati, Italy \\ ${ }^{b}$ Department of Basic and Applied Science for Engineering (SBAI) Sapienza, University of Rome, \\ Via A. Scarpa 14, 00161 Roma, Italy \\ ${ }^{c}$ Paul Scherrer Institute (PSI), \\ 5234 Villigen, Switzerland \\ E-mail: David.Alesini@lnf.infn.it
}

ABSTRACT: In this paper we illustrate the tuning procedure we implemented to tune the traveling wave structures designed and constructed for the SPARC photo-injector energy upgrade. The procedure has been derived from that proposed and adopted for the CLIC structures at CERN (J. Shi et al., Tuning of clic accelerating structure prototypes at Cern, Proc. of LINAC 2010, Tsukuba, Japan, 2010). In the first part of the paper we go through the analytical formulas also illustrating and discussing an algorithm to tune the input and output couplers. A detailed description of the measurements we have done before and after the tuning of the SPARC C-band structure prototype is then illustrated.

KEYWORDS: Acceleration cavities and magnets superconducting (high-temperature superconductor; radiation hardened magnets; normal-conducting; permanent magnet devices; wigglers and undulators); Accelerator Subsystems and Technologies; Accelerator Applications

\footnotetext{
${ }^{1}$ Corresponding author.
} 


\section{Contents}

1 Introduction 1

2 Tuning method description 3

2.1 Local reflection coefficients due to cell imperfections 3

$\begin{array}{ll}2.2 & \text { Tuning procedure for regular cells }\end{array}$

$\begin{array}{lll}2.3 & \text { Tuning of the input and output couplers } & 7\end{array}$

3 Tuning equipment description 9

4 Tuning of C-band TW structure prototype 9

4.1 Electric field measurement 9

$\begin{array}{ll}4.2 & \text { Tuning procedure } \\ \end{array}$

5 Conclusions $\quad 15$

A Theoretical analysis of the reflection due to cells de-tuning 16

\section{Introduction}

Traveling wave (TW) accelerating structures are used for relativistic electron acceleration [1, 2]. They are disc loaded structures basically constituted by an input coupler, several accelerating cells (in general of the order of one hundred), and an output coupler. The input coupler converts the $\mathrm{RF}$ waveguide mode into the accelerating mode $\mathrm{TM}_{01}$-like avoiding power reflections. The electromagnetic field propagates into the structure through the cells with a phase velocity equal to the beam velocity (c), accelerating the beam itself. The output coupler converts the residual power not dissipated into the metallic wall of the structure or not absorbed by the beam, into the waveguide mode. This residual power is, in general, dissipated into an RF load in order to avoid reflections. Power reflections can, in fact, give standing wave patterns into the accelerating structure that can perturb the beam dynamics, enhance the peak field and damage the RF power source.

These structures are finely designed and realized, nonetheless the technology used to produce the pieces of a traveling wave section has a limited precision in producing identical cells. Very high precisions in the cell manufacture are not economical and can give a strong increase in the cost of the structure. Moreover also the brazing process can give variations of structure dimension that have to be compensated or corrected. Since the resonant frequencies of the cells depend on their geometrical parameters, errors in their dimensions introduce several effects that degrades the performance of the whole structure.

On the other hand, by relaxing the tolerances on the fabrication techniques, it is possible to reduce the total cost considerably, while the geometrical imperfections can be compensated through 
the process of tuning for a relatively small incremental cost. Several tuning procedure for TW structures have been proposed and implemented [3]-[6]. The process consists in the determination of the frequency error of each cell and in the correction of such error by deformation. The several tuning procedures, basically, differ in the technique used to evaluate this frequency errors. Some of the proposed solutions [3] are based on a detuning of the structure with insertions of a metallic plunger progressively pulled through the structures itself. More recent technique [4]-[6] are indeed based on accelerating field profile measurements using perturbations techniques [7]. This avoid the insertion, on the disc loaded structure, of possible dangerous metallic plungers that can hit the irises damaging the structure itself. The final phase shift per cavity, to avoid a significative reduction of the total accelerating gradient in the structure, can be fixed in our case as \pm 4 deg while the overall cumulative phase shift have to be less than $\pm 5 \mathrm{deg}$. These tollerance values can be easily found with a simple analysis of the final accelerating gradient introducing random errors in the cells within these specified limits.

In this paper we discuss the tuning of the SPARC C-Band prototype according to the procedure recently adopted at CERN [6] in the context of the X-band accelerating structure development program for the CLIC study. The SPARC energy will be upgraded from $170 \mathrm{MeV}$ to more than $240 \mathrm{MeV}$ by replacing a low gradient S-band traveling wave section with two C-band TW constant impedance structures. The implementation of this system at SPARC has been proposed to explore the C-Band acceleration combined with a S-Band injector that, at least from beam dynamics simulation, seems to be very promising for the achievable beam quality. The C-Band acceleration allows, in principle, obtaining higher gradients and thus a shorter LINAC, and also this point can be attractive for future upgrades of the SPARC photo-injector. An important requirement, for the new system, is to reach an average accelerating gradient $>35 \mathrm{MV} / \mathrm{m}$, with a total energy gain in the two sections $>100 \mathrm{MeV}$. A detailed description of the design criteria of such structures is given in [8].

Before the construction of the final structures a prototype with a reduced number of cells has been realized. The C-band prototype is a traveling wave constant impedance section, composed by 22 regular cells, coupled each other by irises, and an input and an output coupler, in a $54 \mathrm{~cm}$ length. The goals of this prototype were the test of all design and construction procedure and the test of the structure at high power at KEK (Japan) in the framework of the ongoing collaboration between LNF and KEK [9]. The tuning of this prototype has been of fundamental importance to deeply understand the tuning mechanism and to check the design and the fabrication procedure.

In the first section of the paper we review and integrate, with analytical calculations, the results got in [6] to implement an efficient algorithm of tuning. The review of the algorithm consists in a full development of the calculations highlighting the several approximations of the obtained tuning formulae. The clue of the algorithm is an iterative system whose solution can be, in principle, approximated in several ways and in different steps. A first order solution gives two possible simple formulae for the calculations of the local reflection coefficients. One of this is that obtained in [6] but also the second formula can be, in principle, used giving similar results. The description of the algorithm and the final formula obtained for the iterative system open, also, several possibilities to further develop the algorithm using, for example, a second order approximation for the local reflection coefficient calculations. 
The integration of the algorithm consists, on the other hand, in the develop of a tuning method to compensate the reflections of the output couplers. The technique based on the local reflections calculation and compensation allows, indeed, to tune the central cells of the structure but not the reflections given by the output coupler. These reflections have to be properly compensated to avoid phase advance mismatches between cells in the last part of the accelerating structure and enhancement of the electric field due to the superposition of the forward and reflected waves.

The algorithm we present allows to compensate such reflection by a proper tuning of the cells near the output coupler. In this way, we are able to provide a complete tuning procedure which has been successfully applied to the C-band traveling wave section prototype. The experimental measurements and the achieved results are illustrated and discussed in the second and third section.

\section{Tuning method description}

A generic traveling wave section is composed by several cells (identical in constant impedance structure) strongly coupled each other. Every cell of the section can be considered as a pillbox cavity, with its own resonant frequency.

At the working frequency of the structure the phase difference between adjacent cells is chosen to assure synchronicity with the beam. The $2 \pi / 3$ phase advance per cell usually gives the best compromise between structure length, group velocity (filling time), overall dissipation and efficiency [3].

A variation of the cell dimensions with respect to the nominal ones (or equivalently a frequency detuning of the cells) causes several correlated effects:

- a shift of the phase advance per cell;

- a change in the phase velocity $v_{p h}$ of the TW field;

- power reflections at the detuned cell position and thus a generation of a stationary wave.

The first two effects can perturb or compromise the synchronization of the TW field with the accelerated beam while the third one can also introduce local field enhancement limiting the overall accelerating gradient. For reader convenience, we recall that the final phase shift per cavity, to avoid a significative reduction of the total accelerating gradient in the structure, can be fixed in our case as \pm 4 deg while the overall cumulative phase shift have to be less than $\pm 5 \mathrm{deg}$.

In this section we are going to critically review the method implemented and tested at CERN for tuning the CLIC-G traveling wave prototype [6], adding the procedure to tune the coupler as well.

\subsection{Local reflection coefficients due to cell imperfections}

According to ref. [6], if we refer to the whole traveling wave structure as a lossless transmission line, the longitudinal electric field in the $n$-th cell is expressed by the sum of a progressive wave $A$ (traveling from input to output coupler) and a regressive wave $B$ (traveling from output to input coupler). The field in the $n$-th cell is the superposition of two waves

$$
I_{n}=A_{n}+B_{n}
$$




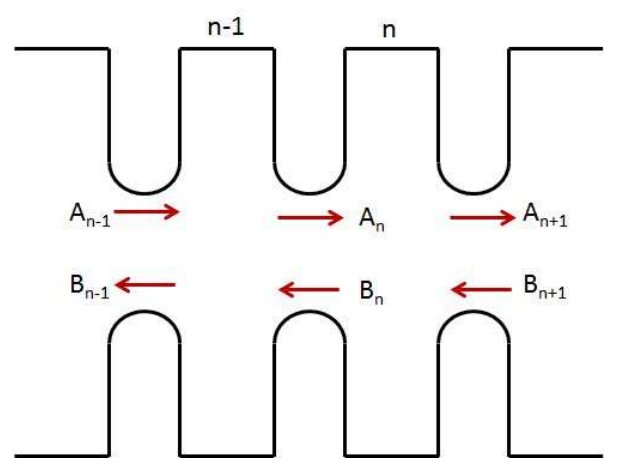

Figure 1. Forward wave (A) and backward wave (B) in the n-th cell of a section.

where $A_{n}\left(B_{n}\right)$ is the progressive (regressive) wave in the $n$-th cell. In the ideal case $B_{n}=0$ for any $n$. The index $n$ ranges from $n=1 \ldots N$; the cell $n=1$ is the input coupler while the cell $n=N$ is the output coupler.

Let us now consider a generic cell of the structure and the two waves, as given in figure 1 where $A_{n}, B_{n}$ are represented at the iris positions. According to this model, the single cell can be described as a two port network with its scattering matrix $S[10]$ whose properties are based on the following hypothesis:

- each cavity is assumed lossless (i.e. scattering matrix is unitary);

- each cell does not contain nonreciprocal material (i.e. scattering matrix is symmetrical);

Therefore the scattering matrix $S_{n}$ of the $n$-th cell can be written as

$$
S_{n}=\left[\begin{array}{cc}
\Gamma_{n} & \sqrt{1-\left|\Gamma_{n}\right|^{2}} e^{-j \varphi_{n}} \\
\sqrt{1-\left|\Gamma_{n}\right|^{2}} e^{-j \varphi_{n}} & \Gamma_{n}
\end{array}\right] .
$$

The scattering parameter $\Gamma_{n}$ can be considered a "local" reflection coefficient. $\varphi_{n}$ is the phase advance per cell and, for regular cells $n=2 \ldots N-1$, it is given by the sum of two terms:

$$
\varphi_{n}=\varphi+\xi_{n} .
$$

The first term $\varphi$ is the nominal phase advance per cell, while the second term $\xi_{n}$ is the variation of the phase advance due to detuning effects. In each cell the progressive and regressive waves are then related to the cell scattering matrix by the following relations:

$$
\left[\begin{array}{c}
B_{n} \\
A_{n+1}
\end{array}\right]=\left[\begin{array}{cc}
\Gamma_{n} & \sqrt{1-\left|\Gamma_{n}\right|^{2}} e^{-j \varphi_{n}} \\
\sqrt{1-\left|\Gamma_{n}\right|^{2}} e^{-j \varphi_{n}} & \Gamma_{n}
\end{array}\right]\left[\begin{array}{c}
A_{n} \\
B_{n+1}
\end{array}\right] .
$$

The electric field in each cell is related to $A_{n}$ and $B_{n}$ by eq. (2.1) and, in principle, it can be measured. Moreover if the load connected to output coupler is ideal, then $B_{N+1}=0$ while the (measurable) reflection coefficient at the structure input port is

$$
\Gamma_{\text {global }}=\frac{B_{1}}{A_{1}}
$$


The non linear system given by eqs. (2.1), (2.4), (2.5) has $3 N+1$ complex equations and $3 N+1$ complex unknowns $\left(A_{1} \ldots A_{N+1}, B_{1} \ldots B_{N}, \Gamma_{1} \ldots \Gamma_{N}\right)$ plus $N$ real unknowns $\left(\varphi_{1} \ldots \varphi_{N}\right)$. Therefore such system can not be solved.

Nevertheless the system can be simplified for regular cells $(n=2 \ldots N-1)$ since we can assume

$$
\varphi_{n} \approx \varphi, \quad\left|\Gamma_{n}\right| \ll 1 \Rightarrow \sqrt{1-\left|\Gamma_{n}\right|} \approx 1 .
$$

Therefore the system reads

$$
\left\{\begin{array}{l}
B_{n}=\Gamma_{n} A_{n}+e^{-j \varphi} B_{n+1}, \\
A_{n+1}=e^{-j \varphi} A_{n}+\Gamma_{n} B_{n+1}, \\
I_{n}=A_{n}+B_{n}
\end{array}\right.
$$

for $n=2 \ldots N-1$.

For reader convenience, we can write the system of eq. (2.7) for two adjacent cells

$$
\left\{\begin{array}{l}
B_{n}=\Gamma_{n} A_{n}+e^{-j \varphi} B_{n+1}, \\
A_{n+1}=e^{-j \varphi} A_{n}+\Gamma_{n} B_{n+1}, \\
I_{n}=A_{n}+B_{n} \\
B_{n-1}=\Gamma_{n-1} A_{n-1}+e^{-j \varphi} B_{n}, \\
A_{n}=e^{-j \varphi} A_{n-1}+\Gamma_{n-1} B_{n}, \\
I_{n-1}=A_{n-1}+B_{n-1}
\end{array}\right.
$$

even in such a system, the number of unknowns is greater than the number of equations and therefore the system can not be solved in a closed form.

A recursive solution is anyway feasible. The first step of the iteration assumes $\Gamma_{n}=\Gamma_{n-1}=0$ which results

$$
\begin{aligned}
A_{n} & =\frac{I_{n-1}-I_{n} e^{-j \varphi}}{2 j \sin \varphi}, & B_{n} & =\frac{I_{n-1}-I_{n} e^{i \varphi}}{-2 j \sin \varphi}, \\
A_{n+1} & =\frac{I_{n}-I_{n+1} e^{-j \varphi}}{2 j \sin \varphi}, & B_{n+1} & =\frac{I_{n}-I_{n+1} e^{i \varphi}}{-2 j \sin \varphi} .
\end{aligned}
$$

Including eqs. 2.9 into the first equation of system 2.8 we obtain

$$
\Gamma_{n}=\frac{B_{n}-B_{n+1} e^{-j \varphi}}{A_{n}}
$$

thus

$$
\Gamma_{n}=\frac{-I_{n-1}+2 I_{n} \cos \varphi-I_{n+1}}{I_{n-1}-I_{n} \exp (-j \varphi)} .
$$

Similarly, including eqs. 2.9 into the second equation of system 2.8 we then get

$$
\Gamma_{n}=\frac{A_{n+1}-A_{n} e^{-j \varphi}}{B_{n+1}}
$$

thus

$$
\Gamma_{n}=\frac{-I_{n-1}+2 I_{n} \cos \varphi-I_{n+1}}{-I_{n} \exp (j \varphi)+I_{n+1} \exp (j 2 \varphi)} .
$$

It is easy to verify that eq. (2.11) and eq. (2.13) are equal if the reflected wave $B_{n}$ is much smaller than the incident wave $A_{n}$. Equation (2.11) (or eq. (2.13)) allows inferring the local reflection 
coefficient $\Gamma_{n}$ for the regular cells by measuring the field in the cells $n-1, n$ and $n+1$. Wave reflections at the cell are due to cell imperfections and can be compensated by deformation of the outer wall of the cells themselves, as described in the next section.

\subsection{Tuning procedure for regular cells}

The theoretical model given in appendix A shows that the local reflection coefficients $\Gamma_{n}$ due to cells detuning should be purely imaginary. That results shows also that the imaginary part of $\Gamma_{n}$ can be compensated (i.e. brought to zero) by changing the resonant frequency $f_{\text {res }}$ of the cell, i.e. by properly tuning the cells for example locally deforming the outer cell wall. Such simplified model allows also to predict the sign of the resonant frequency shift that should be applied to correctly tune the cell. Indeed for each regular cell $n$, eqs. (2.11) and (2.13) read

$$
\Gamma_{n}=j Q_{0, n}^{\prime} \frac{\Delta f_{n}}{f_{R F}}
$$

where $\Delta f_{n}=f_{\text {res, } n}-f_{R F}$ with $f_{\text {res }, n}$ being the resonant frequency of the $n$-th cell and

$$
Q_{0, n}^{\prime}=\frac{c \varphi_{n}}{v_{g, n}}
$$

where $v_{g, n}$ is the group velocity of field in the $n$-th cell and $\varphi_{n}$ is the phase advance per cell that we will assume constant.

On the other hand it also easy to demonstrate [6] that a variation of the local reflection coefficient $\left(\left|\Delta \Gamma_{n}\right|\right)$ is related to the variation of the global reflection coefficient at the structure input port $\left(\left|\Delta \Gamma_{\text {global }}\right|\right)$ since

$$
\left|\Delta \Gamma_{\text {global }}\right|=e^{-2 \alpha(n)}\left|\Delta \Gamma_{n}\right|
$$

where

$$
\alpha(n)=\sum_{i=1}^{n-1} \alpha_{i} D
$$

and $\alpha_{i}$ is the $i$-th cell attenuation constant and $D$ is the cell length. For a constant impedance structure we have that $\alpha_{i}=\alpha$ and thus $\alpha(n)=(n-1) \alpha D$.

Therefore to tune the regular cells of the structure one can

1. measure the complex field distribution at working frequency using a bead-pull technique [7];

2. calculate the local reflection coefficients $\Gamma_{n}$ of each regular cell with eq. (2.11);

3. predict the deformation to be applied to the cells by eq. (2.14);

4. tune the cells looking at the variation of the modulus of the global reflection coefficient $\left|\Delta \Gamma_{\text {global }}\right|$ according to eq. (2.16);

5. repeat such procedure until the $\Gamma_{n}$ is smaller of the desired value (typically $\left|\Gamma_{n}\right|<0.005$ ). 
For example, if the tuning is performed with tuning screws deforming locally the cell walls in a magnetic field region, the tuning screw should be pushed or pulled outside depending on the sign of local reflection coefficient imaginary part:

$$
\Gamma \approx j \frac{Q_{0}^{\prime} \Delta f}{f_{R F}} \Rightarrow\left\{\begin{array}{l}
\operatorname{Im} \Gamma>0 \text { if } \Delta f>0 \Longleftrightarrow f_{\text {res }}-f_{R F}>0 \Longrightarrow \text { pull } \\
\operatorname{Im} \Gamma<0 \text { if } \Delta f<0 \Longleftrightarrow f_{\text {res }}-f_{R F}<0 \Longrightarrow \text { push }
\end{array} .\right.
$$

If the imaginary part of $\Gamma_{n}$ is negative, due to a negative value of $\Delta f$, the resonant frequency of the cell becomes lower than the working frequency; therefore to correct a detuning in the cell it is necessary to increase the cell resonant frequency. This can be achieved by reducing the volume of the cell by pushing the tuner inside of it. On the contrary, if the imaginary part of $\Gamma_{n}$ has a positive value, resonant frequency of the cell has to be decrease by enlarging its volume pulling the tuner outside of it.

\subsection{Tuning of the input and output couplers}

As already pointed out the illustrated tuning procedure is valid only for regular cells $(n=2 \ldots N-$ 1 ), but not for the input and the output couplers. Indeed the above procedure assumes $\varphi_{n}$ equal to the nominal phase advance per cell $\varphi$ and it needs three field measurements $\left(I_{n}, I_{n-1}, I_{n+1}\right)$ on the adjacent cells to infer the local reflection coefficient $\Gamma_{n}$. For the input (output) coupler, the field $I_{n-1}\left(I_{n+1}\right)$ is not defined and moreover $\varphi_{n} \neq \varphi$. For the same reasons, also the cell adjacent to the couplers do not satisfy the assumptions of the tuning method and they may not be perfectly tuned. On the other hand, after having tuned all regular cells, any residual field error (in modulus and phase) is due to mismatch localized in the output coupler (or in the adjacent one).

Therefore, let us assume that the output coupler introduce some reflection and the reflected wave $B_{N}$ propagates back towards the input coupler. If the cells of the structure were perfectly tuned, the reflected wave at the $(N-i)$-th cell due to the propagation of $B_{N}$ is

$$
B_{N-i}=B_{N} e^{-j i \varphi}
$$

thus by measuring the reflected wave in the $(N-i)$-th cell we can infer the wave $B_{N}$ reflected at the output coupler. In order to increase the accuracy of $B_{N}$ estimation, we may average on $M$ cells that is

$$
\bar{B}_{N}=\frac{1}{M} \sum_{i=1}^{M} B_{N-i} e^{j i \varphi} .
$$

Then, the local reflection coefficient of the coupler cell can be estimated as

$$
\bar{\Gamma}_{N}=\frac{\bar{B}_{N}}{A_{N}}
$$

which has, in general, a real and an imaginary part. $\bar{\Gamma}_{N}$ may have a real part due to errors in irises and coupling windows.

Therefore, according to eq. (2.13), such reflection can not be compensated with tuners in the coupler cell only but one has to use, at least, the tuners of the cell adjacent to the coupler too. In 
this case, it is necessary to consider the output coupler as the ensemble of the cells $N-1$ and $N$ which means, first estimate $\bar{B}_{N-1}$ as the average

$$
\bar{B}_{N-1}=\frac{1}{M} \sum_{i=2}^{M} B_{N-i} e^{j(i-1) \varphi}
$$

then inferring the local reflection coefficient as

$$
\bar{\Gamma}_{N-1}=\frac{\bar{B}_{N-1}}{A_{N-1}} .
$$

To understand how distribute the correction between $N$ and $(N-1)$ cells, one has to solve the following equation:

$$
\bar{B}_{N-1}+A_{N-1} \Gamma_{N-1}+A_{N} \Gamma_{N} e^{-j \varphi}=0
$$

Dividing the first equation for $A_{N-1}$ and assuming that $A_{N} \approx A_{N-1} e^{-j \varphi}$ we get

$$
\bar{\Gamma}_{N-1}+\Gamma_{N-1}+\Gamma_{N} e^{-j 2 \varphi}=0 .
$$

The reflection coefficient $\bar{\Gamma}_{N-1}$ has both real and imaginary part, while, to the first order, $\Gamma_{N-1}$ and $\Gamma_{N}$ are purely imaginary (eq. (A.3)):

$$
\left\{\begin{array}{l}
\Gamma_{N}=j t_{N}, \\
\Gamma_{N-1}=j t_{N-1} .
\end{array}\right.
$$

Substituting them into eq. (2.25), one gets

$$
\bar{\Gamma}_{N-1}+j t_{N-1}+j t_{N} e^{-j 2 \varphi}=0 .
$$

If $\bar{\Gamma}_{N-1}=\alpha+j \beta$, we can separate the complex equation into its real and imaginary part, obtaining a system in two equations and two variables, with solution given by

$$
\left\{\begin{array}{l}
t_{N}=-\frac{\alpha}{\sin (2 \varphi)} \\
t_{N-1}=\frac{\alpha}{\tan (2 \varphi)}-\beta
\end{array} .\right.
$$

The solution represents the imaginary part of the variation of local reflection coefficient that we had to introduce as correction in the $N$ and $N-1$ cells to compensate the coupler mismatch. Concerning the sign of the correction:

- if $t_{N, N-1}$ is negative, we have to decrease the resonance frequency of the cell, pulling its tuner outside;

- if $t_{N, N-1}$ is positive, we have to increase the resonance frequency of the cell, pushing the tuner inside.

At this point is it possible to tune the output coupler and its adjacent cell, observing the effects of the correction as an improvement of the flatness of the field magnitude profile.

After the tuning of the central cells and of the output coupler it is finally possible to tune the input coupler. This tuning is very simple and it is aimed to reduce the global reflection coefficient at the input port. One can proceed changing the tuner position and minimizing the $\left|\Gamma_{\text {global }}\right|$. 


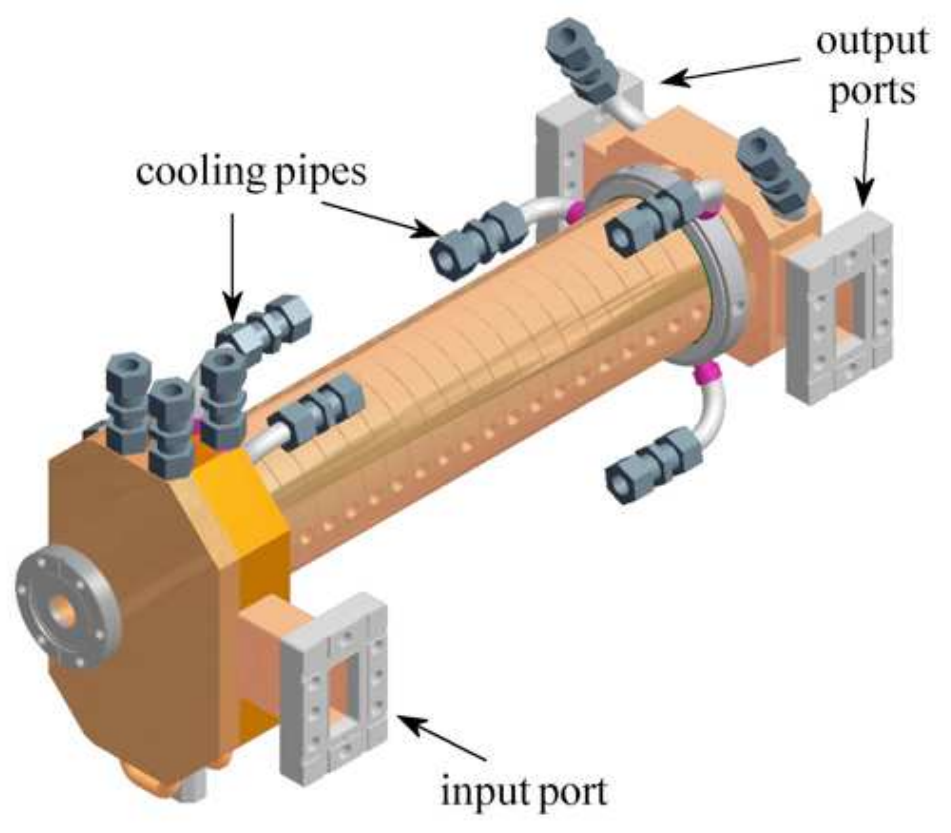

Figure 2. Mechanical design of the C-band prototype.

\section{Tuning equipment description}

The C-band prototype is a traveling wave constant impedance section, composed by 22 regular cells coupled with irises, an input and an output matching coupler, in a $54 \mathrm{~cm}$ length (see figure 2). It has been designed to work at the nominal frequency of $5.712 \mathrm{GHz}$, having a nominal phase advance per cell of $2 \pi / 3$.

The input and output couplers convert the $T E_{10}$ mode of rectangular wave guide in a traveling wave accelerating mode at the design frequency. The external surface of each cell can be locally deformed by the tuners. As shown in figure 3. the cell wall at the tuners location has a reduced thickness allowing a plastic deformation of the wall itself. Such a plastic deformation can be obtained in both directions: the surface can be pushed into the cell by hitting the tuner or can be pulled out with a screw and a dedicated tool as shown in figure 4 . The maximum wall deformation can be of few $10^{-2} \mathrm{~mm}$. Each cell has three tuners separated by $120^{\circ}$ while the couplers have two symmetrical tuners, separated by an angle of $180^{\circ}$.

\section{Tuning of C-band TW structure prototype}

\subsection{Electric field measurement}

For field measurement of the C-band traveling wave section it has been used Steele's non-resonant perturbative method [7].

With the Steele's bead-pull method one can measure amplitude and phase of the electric field on the axis of the structure; measurements of the reflection coefficient $S_{11}$ can be acquired at the 

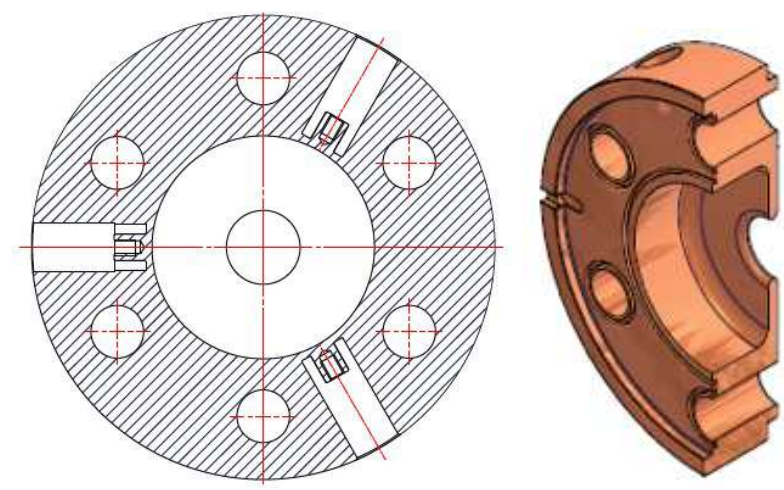

Figure 3. Mechanical drawing of a single cell; the three white spaces are the tuning holes, while the circles are for cooling system allocation.

(a)

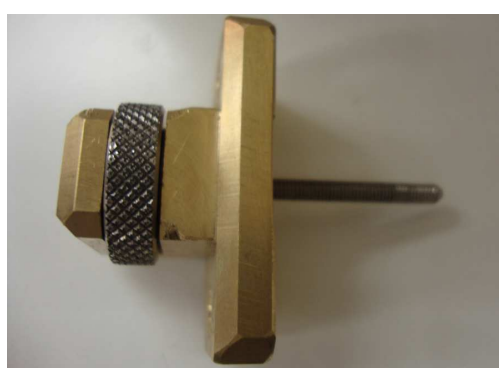

(b)

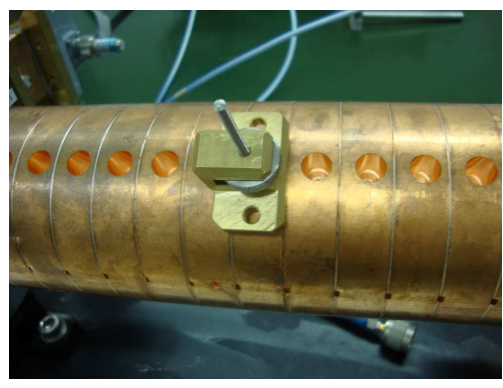

Figure 4. Tuning screw with wheel (a) and tuning screw applied on the section (b).

same frequency with and without a perturbing object placed at the point at which the field parameters have to be determined.

On the axis of the section the magnetic field is zero and the dependence of the reflection coefficient from the electric component of the field is expressed by the following formula $[5,11]$ :

$$
2 P_{i}\left(S_{11 p}-S_{11 u}\right)=-j \omega k E_{a}^{2}
$$

with $P_{i}$ the input power, $S_{11 p}$ the reflection coefficient in the presence of a perturbing object, $S_{11 u}$ the reflection coefficient in the absence of the perturbing object, $k$ a constant that depends on geometry of the object and $E_{a}$ the electric field on the perturbing bead position.

The perturbing object is a pierced cylindrical needle, pasted on a nylon line, of $1 \mathrm{~mm}$ diameter and a $2.1 \mathrm{~mm}$ length, oriented parallel to the axis of the section. The bead can be moved along this axis using a step motor controlled. During the bead-pull measurement the motor wrap and unwrap the line around a reel as the bead movement covers $56 \mathrm{~cm}$ in left direction and then other $56 \mathrm{~cm}$ in the opposite direction to come back to initial position.

To perfectly center the nylon line into the section hole two pierced teflon dishes as been realized and installed at the input and output of the section. In this way a nylon line of $0.2 \mathrm{~mm}$ diameter has to pass into a hole of $0.4 \mathrm{~mm}$ diameter. All the measurements has been acquired with the Vector Network Analyzer HP8753.

Before starting field measurement necessary to tune the structure, we have calculated the the working frequency $\tilde{f}_{R F}$ of the structure in the condition of temperature and pression of the labora- 

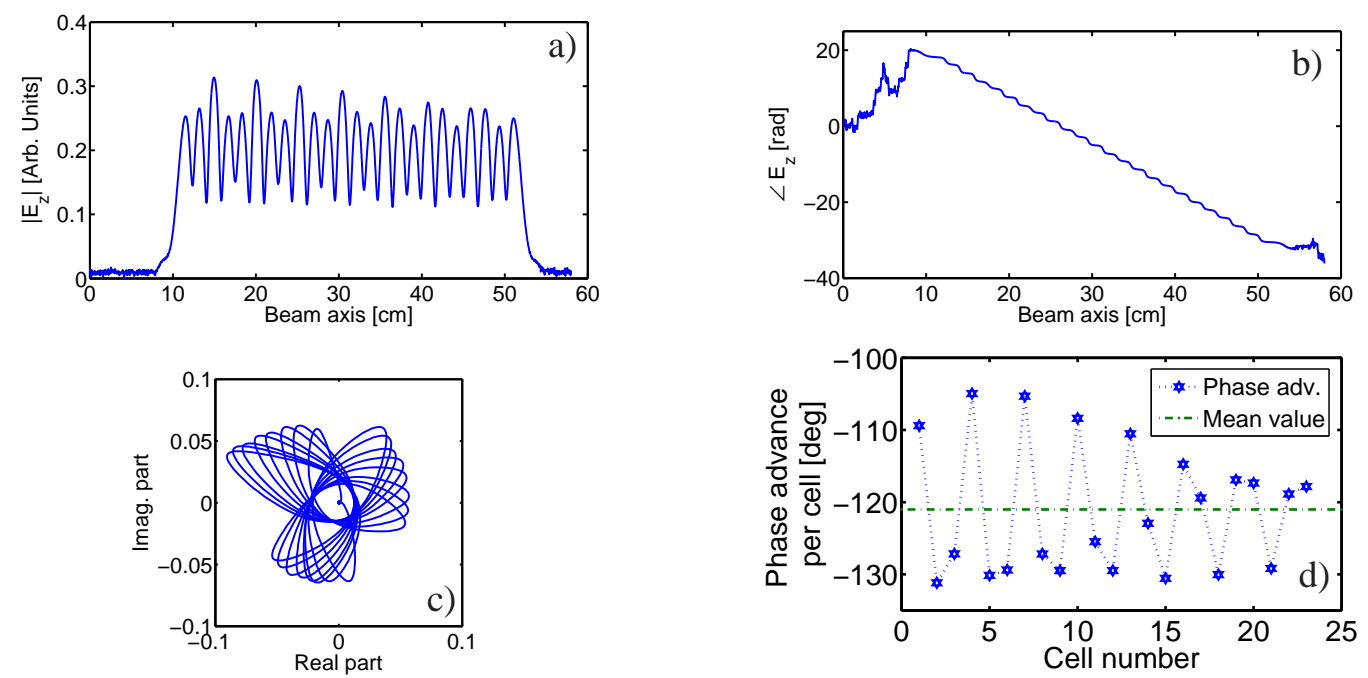

Figure 5. RF measurement results before tuning; the average phase advance is -121.1 (1.9) deg.

tory, using the following formula [5]:

$$
\tilde{f}_{R F}=\frac{f_{R F}}{\left(1+1.7 \cdot 10^{-5}\left(T_{\mathrm{amb}}-T_{\mathrm{prog}}\right)\right)} \frac{1}{\sqrt{\varepsilon}},
$$

where $f_{R F}=5.712 \mathrm{GHz}$ is the working frequency of the structure at the nominal working temperature of $T_{\text {prog }}=30^{\circ} \mathrm{C}$.

In order to calculate the relative dielectric constant $\varepsilon$ and pressure $P_{0}$ we can use the following formulae:

$$
\begin{aligned}
\varepsilon & =1+P_{\mathrm{air}} / T_{\mathrm{amb}}\left(211+P_{0} H_{\mathrm{air}} / P_{\mathrm{air}}\left(10160 / T_{\mathrm{amb}}-0.294\right)\right) \cdot 10^{-6} \\
P_{0} & =10^{\left(7.45\left(T_{\mathrm{amb}}-273\right) /\left(T_{\mathrm{amb}}-38.3\right)+0.656\right)}
\end{aligned}
$$

where $H_{\text {air }}$ is the air humidity, $P_{\text {air }}$ the air pressure and $T_{\text {amb }}$ is the temperature of the structure during measurements.

In figure 5 are shown bead-pulling measurement results for the section before the tuning.

The structure detuning can be highlighted by the RF phase diagram (figure 5.c) in which the three "petals" are not superimposed (the Smith Chart plot corresponds to the measured quantity of eq. (4.1) proportional to $E^{2}$ ). It corresponds to a difference in the phase advance per cell (figure 5.d) and to a field "unflatness" (figure 5.a).

We have then calculated the local reflection coefficients for regular cells. The result is given in figure 6 where the real and imaginary part of the local reflection coefficient have been calculated. In principle, only the imaginary part can be compensated with tuning.

All regular cells (2-23) have a negative imaginary part of the local reflection coefficient. Therefore the tuning must "push up" the imaginary part of the local reflection coefficient of each single cell, to move it as near as possible to zero. Thus, for all the regular cells, we begin the tuning pushing the tuners inside the structure, in order to reduce cells volume, and increase the resonant frequency of each cell, according to eq. (2.18). 


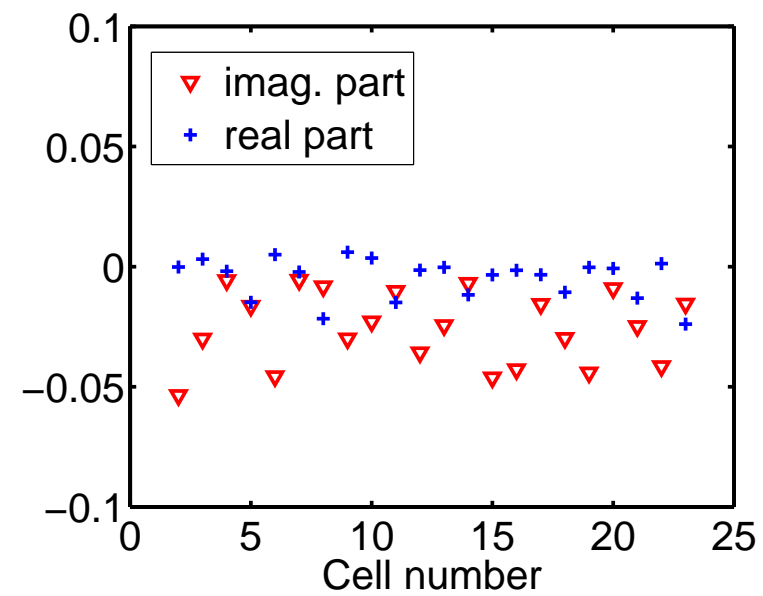

Figure 6. Imaginary part of the local reflection coefficients of the cells before starting tuning.

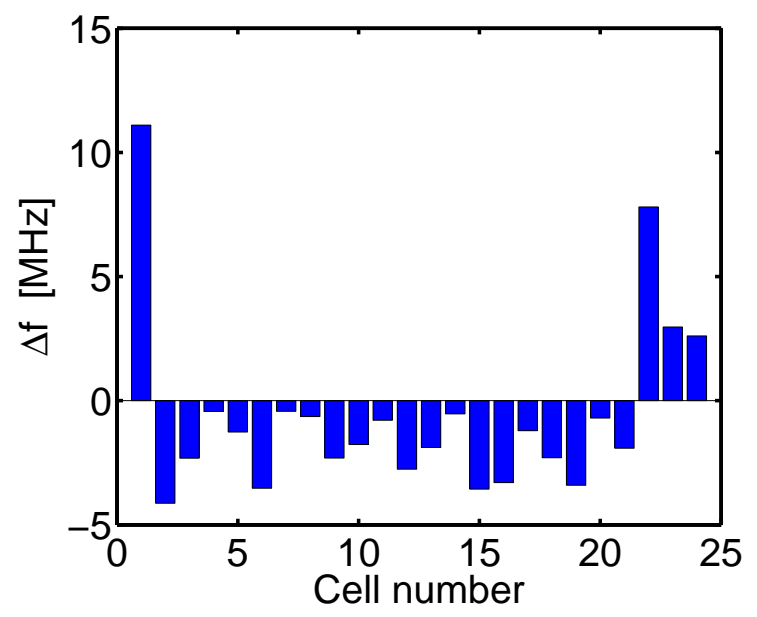

Figure 7. Detuning of the regular cells.

From the local reflection is also possible to calculate the detuning of each cell by applying eq. (2.14). The results are given in figure 7 and show an average detuning of the order of few $\mathrm{MHz}$ corresponding to mechanical errors on the outer radius of about few $0.01 \mathrm{~mm}$.

The measured global reflection coefficient at the input port is given in figure 8 and, at the working frequency was about $-21 \mathrm{~dB}$.

\subsection{Tuning procedure}

The regular cells of the structure have been tuned according to the procedure discussed in section 2.2. The results after the tuning in terms of electric field measurements and local reflection coefficients are given in figure $9-10$ respectively.

For the output coupler it has been applied the correction method discussed in section 2.3 assuming cell 23 part of the output coupler. Concerning the input coupler we have slightly moved the tuners of the matching cell seeking for the minimum global reflection coefficient at the input 


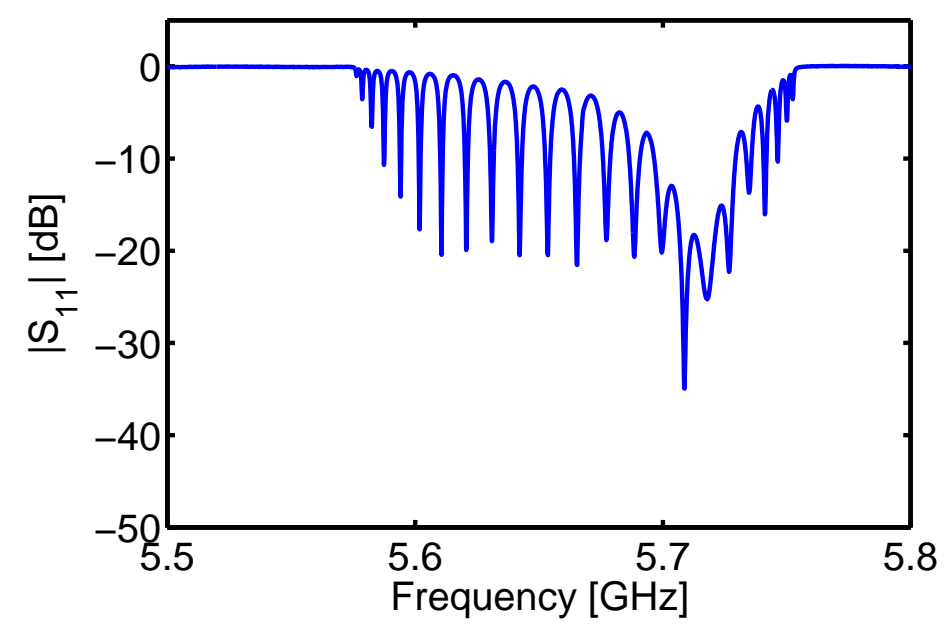

Figure 8. Reflection coefficient versus frequency at the input port before tuning.
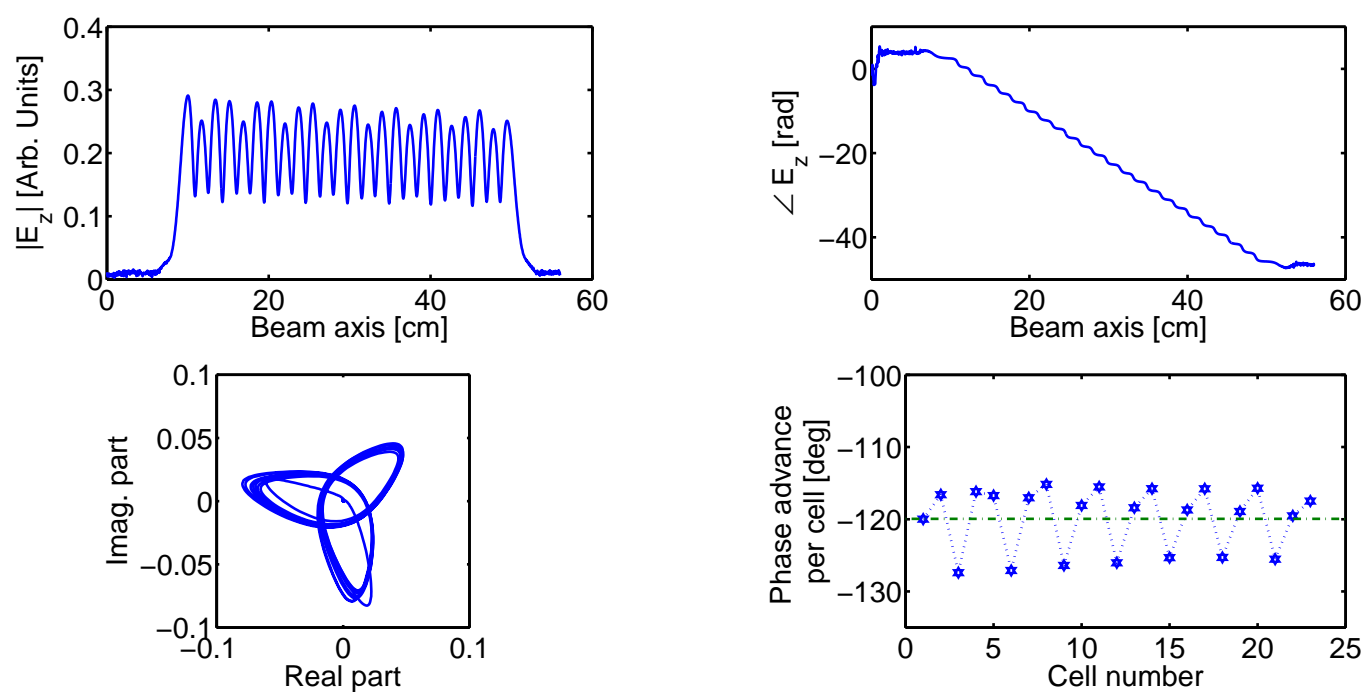

Figure 9. RF measurement results after a first tuning of the 20 central cells of the structure; the average phase advance is $-119.94(0.92) \mathrm{deg}$.

port. The final results in terms of electric field measurements and global reflection coefficients are given in figure 11-12 respectively. The measured global reflection coefficient at the working frequency moved from $-21 \mathrm{~dB}$ (before tuning) to $-35 \mathrm{~dB}$.

The final local reflection coefficients are given in figure 13. From this plot, it is possible to note that the local reflection coefficients of the last three cells is non negligible, because the last regular cell (cell 23) has been used to compensate the output coupler mismatch. From the same plot, it is also possible to note that there are not negligible real parts of the local reflection coefficients giving the residual phase advance per cell errors of figure 11. The residual real part may be due to errors on the coupling irises between few cells. Nevertheless such imperfections have negligible effects in the beam acceleration. 


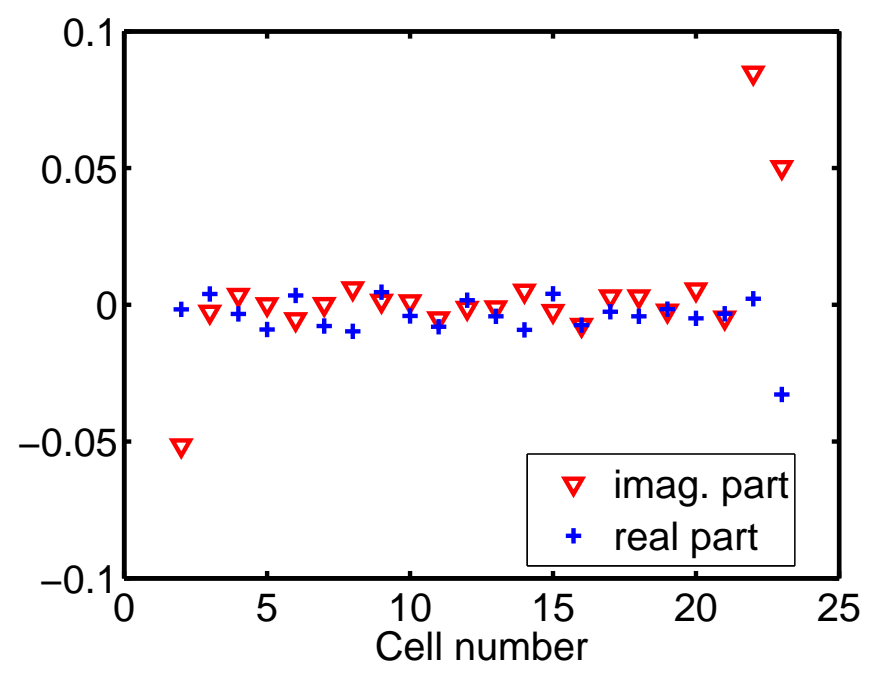

Figure 10. Real and imaginary part of the local reflection coefficient after tuning for each regular cell.
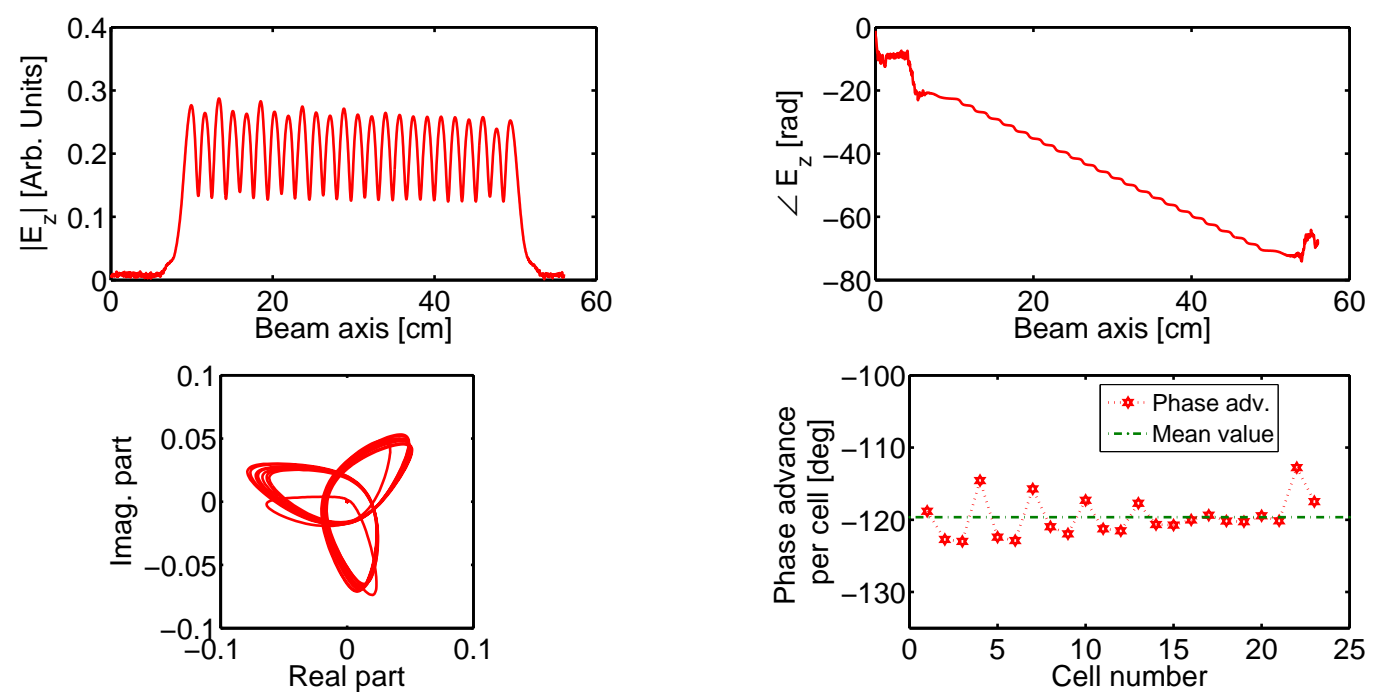

Figure 11. RF measurement results after tuning; the average phase advance is $-119.65(0.56) \mathrm{deg}$.

Comparing figure 5 and figure 11, one can see the improvement in the electric field phase and magnitude after the tuning. The filed flatness goes from about $8 \%$ to less than $4 \%$, while the phase advance per cell changed from more than \pm 10 deg to less than \pm 5 deg in the first 10 cells and less $\pm 2 \mathrm{deg}$ in the last part of the structure. The overall cumulative phase shift is $<5 \mathrm{deg}$. These results shown that the structure specifications have been reached. This improvements are related to the reduction of the difference between the resonant frequency of a cell and working frequency of the section (i.e. the frequency detuning) as shown in figure 14 . 


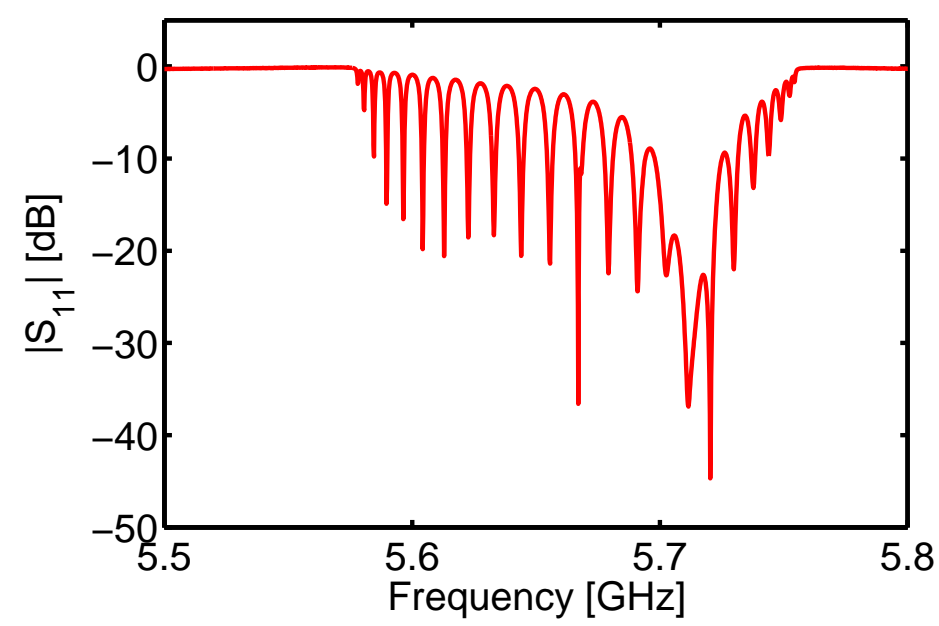

Figure 12. Global reflection coefficient after tuning.

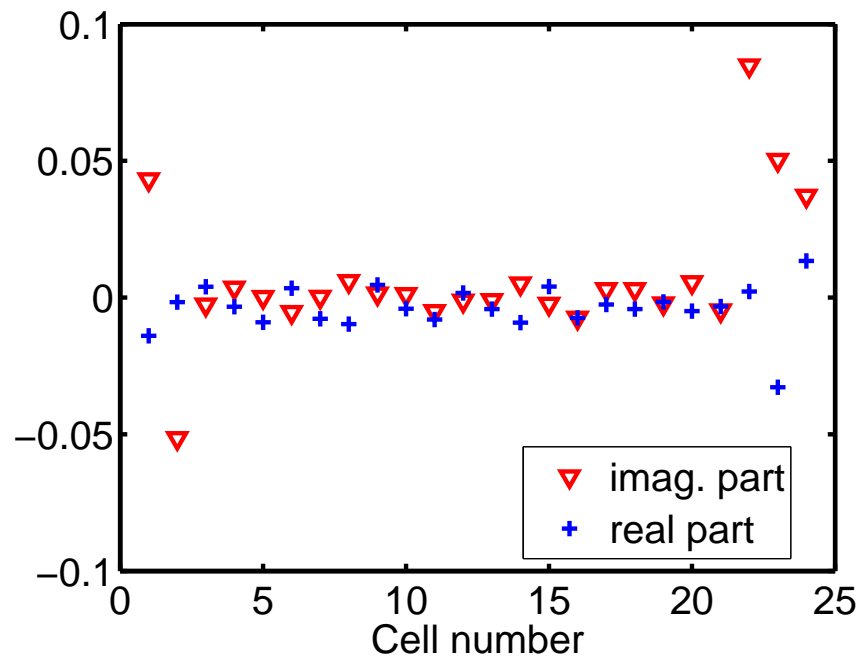

Figure 13. Real and imaginary part of the local reflection coefficient for all the cells (regular ones and couplers).

\section{Conclusions}

The present work focused on a method for tuning the traveling wave C-Band structures for the SPARC photo-injector energy upgrade. The feasibility study of the prototype is the first step towards the realization of two new C-band sections that will bring SPARC energy from the current $170 \mathrm{MeV}$ to more than $240 \mathrm{MeV}$. For these reasons the tuning procedure has been carefully implemented on a prototype with a reduced number of cells. For tuning the section we followed the same method successfully implemented and tested at CERN for correcting errors in the CLIC-G traveling wave prototype [6]. 


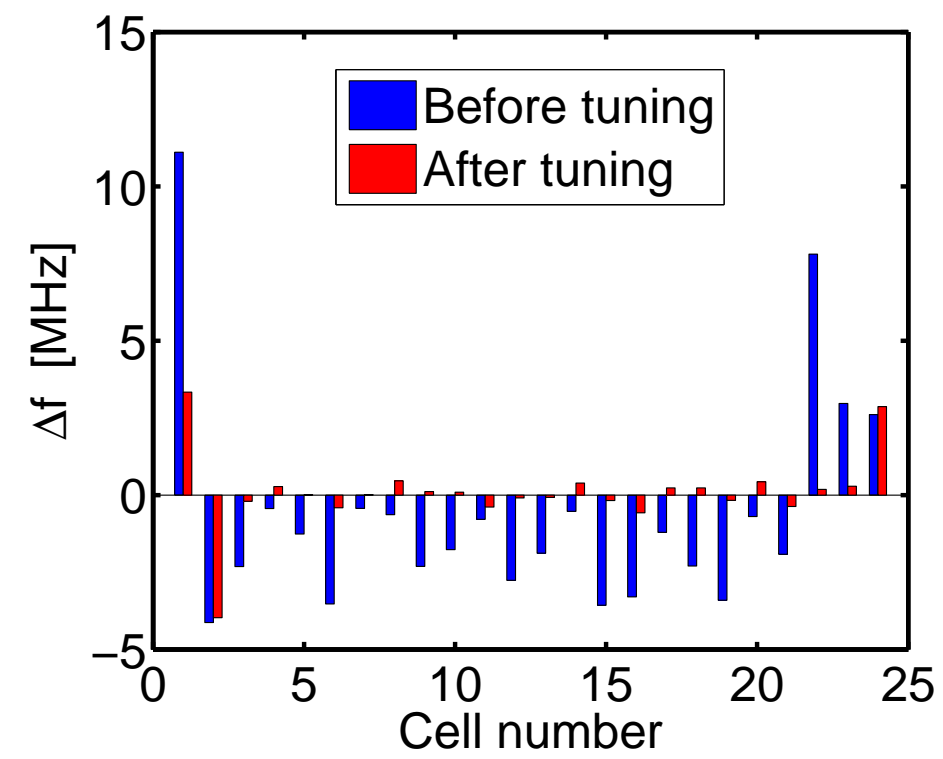

Figure 14. Comparison between frequency detuning of the cells before and after tuning.

In the paper we have derived all the analytical formulas highlighting the several approximations and we have also proposed a procedure to correct errors in the input and output couplers. The whole process has been implemented to tune the C-Band prototype and lead us to a significant improvement of the performances of the structure. In particular the reflection coefficient at the input coupler decreases consistently (about $14 \mathrm{~dB}$ ), getting a final value of $-35 \mathrm{~dB}$, the field flatness goes from about $8 \%$ to less than $4 \%$, while the phase advance per cell changed from more than \pm 10 deg to less than \pm 5 deg in the first 10 cells and less \pm 2 deg in the last part of the structure.

A residual detuning of the cell (and therefore a field flatness unbalance) is still visible and it was not possible to compensate it. Possible reasons of these small residual errors have been discussed.

\section{Acknowledgments}

The research leading to these results has received funding from the European Commission under the FP7-INFRASTRUCTURES-2010-1/INFRA-2010-2.2.11 project TIARA (CNI-PP). Grant agreement no 261905 .

\section{A Theoretical analysis of the reflection due to cells de-tuning}

A traveling wave section is composed by several cells coupled each other by irises. Each cell in the section can be considered as a pillbox cavity having an input and an output ports strongly coupled with the cell itself. 


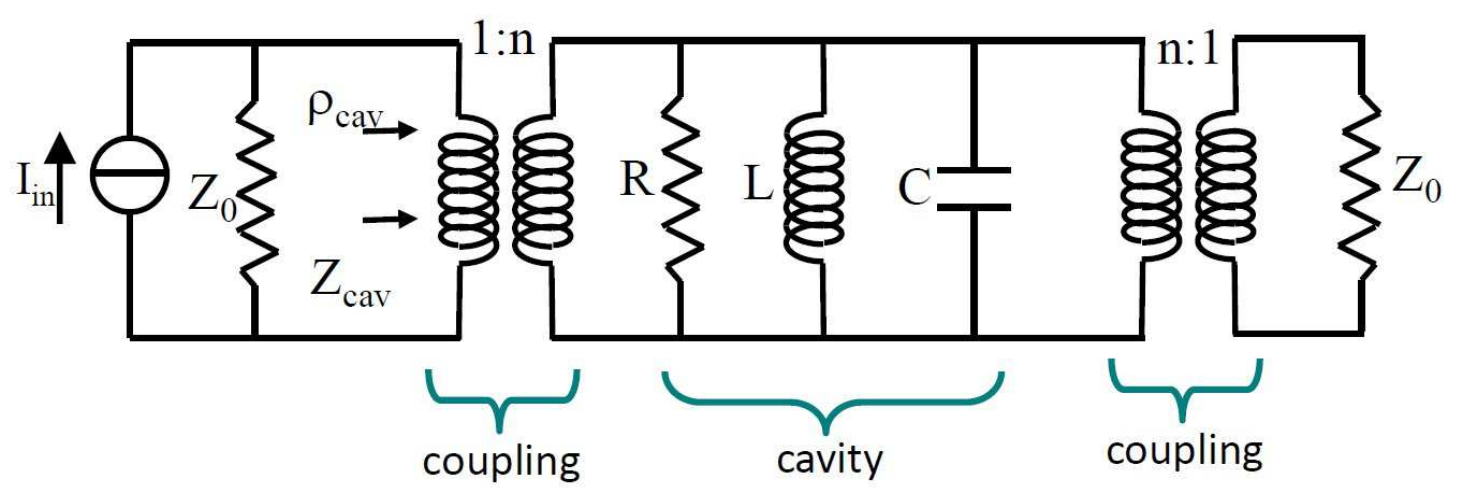

Figure 15. Circuital model of a single cell in a TW structure.

The circuital model is given in figure 15. If the coupling coefficients $\beta_{\text {coupl }}=R /\left(n^{2} Z_{0}\right) \gg 1$, it is easy to demonstrate [12] that the reflection coefficient at the input port is given by

$$
\Gamma=\frac{Z_{\mathrm{cav}}-Z_{0}}{Z_{\mathrm{cav}}+Z_{0}} \approx-j \frac{Q_{0}^{\prime} \delta}{2+j Q_{0}^{\prime} \delta} .
$$

$Q_{0}^{\prime}$ is the loaded quality factor of the resonant mode and $\delta$ is given by

$$
\delta=\frac{f_{R F}}{f_{\text {res }}}-\frac{f_{\text {res }}}{f_{R F}} \approx-2\left(\frac{f_{\text {res }}-f_{R F}}{f_{R F}}\right)=-2 \frac{\Delta f}{f_{R F}},
$$

being $f_{\text {res }}$ the resonant frequency of the cell mode and $f_{R F}$ the working frequency. Since, as demonstrated in the following, $Q_{0}^{\prime}$ is of the order of one hundred, for small cell detuning $\left(\Delta f / f_{R F} \ll 1\right)$ we have then

$$
\Gamma \approx \frac{2 j Q_{0}^{\prime} \Delta f / f_{R F}}{2-j 2 Q_{0}^{\prime} \Delta f / f_{R F}}=\frac{2 j Q_{0}^{\prime} \Delta f / f_{R F}\left(2+j 2 Q_{0}^{\prime} \Delta f / f_{R F}\right)}{4+4\left(Q_{0}^{\prime} \Delta f / f_{R F}\right)^{2}} \approx j Q_{0}^{\prime} \frac{\Delta f}{f_{R F}} .
$$

The loaded quality factor $Q_{0}^{\prime}$ of the cell is given by

$$
Q_{0}^{\prime}=\frac{\omega W}{P_{w}+P_{f}}
$$

where $W$ is the energy stored in the cell, $P_{w}$ is the RF power dissipated on the cell walls and $P_{f}$ is the power flow to the adjacent cell. The power flow is given also by

$$
P_{f}=w v_{g}
$$

where $v_{g}$ is the group velocity of the TW mode and $w$ is the stored energy per unit of length ( $w=W / D$, D being the cell length). Therefore, from previous equations, since for TW structures $P_{f} \gg P_{w}$

$$
Q_{0}^{\prime} \approx \frac{\omega W}{P_{f}}=\frac{\omega D}{v_{g}}=\frac{c \varphi}{v_{g}}
$$

where $\varphi$ is the phase advance per cell and $\omega D / c=\varphi . Q_{0}^{\prime}$ is, in general, of the order of one hundred and thus a frequency detuning $\Delta f / f_{R F}$ in the $10^{-3}$ range gives $|\Gamma|$ of the order of 0.1 . Equation (A.3) shows also that a cell detuning gives a purely imaginary reflection coefficient. 


\section{References}

[1] T. Wangler, Principles of RF Linear Accelerators, John Wiley \& Sons, New York, U.S.A. (1998).

[2] P.M. Lapostolle, Accelerators, North Holland Publishing Company, Amstrerdam, Netherlands (1998).

[3] R.B. Neal general e.d, The Stanford two-mile accelerator, Stanford University, New York, U.S.A. (1968).

[4] T. Khabiboulline, M. Dohlus and N. Holtkamp, Tuning of 50-cell constant gradient s-band traveling wave accelerating structure by using a non resonant perturbation theory, Technical report, Desy M-95-02 (1995).

[5] T. Khabiboulline, V. Puntus and M. Dolhlus, A new tuning method for traveling waves structure, in proceedings of 1995 IEEE Particle Accelerator Conference, Dallas, Texas, U.S.A., 1-5 May 1995.

[6] J. Shi, A. Grudiev, A. Olyunin and W. Wuensch, Tuning of clic accelerating structure prototypes at Cern, in proceedings of LINAC 2010, Tsukuba, Japan (2010);

J. Shi, A. Grudiev, A. Olyunin, W. Wuensch, Tuning of Clic accelerating structure prototypes at CERN, CERN-ATS-2010-210 (2010).

[7] C. Steele, A non resonant perturbation theory, IEEE T. Microw. Theory 14 (1966) 70.

[8] D. Alesini et al., The C-band accelerating structures for SPARC photo-injector energy upgrade, 2013 JINST 8 P05004.

[9] D. Alesini, G. Campogiani, A. Mostacci, L. Palumbo, S. Persichelli and V. Spizzo, Design, fabrication and high power RF test of a C-band accelerating structure for feasibility study of the Sparc photo-injector energy upgrade, in proceedings of IPAC 2011, San Sebastian, Spain (2011).

[10] R.E. Collin, Foundations for microwave engineering, McGraw-Hill (1992).

[11] A. Mostacci, L.Palumbo, R. Da Re, D. Alesini, L. Ficcadenti and B. Spataro, About non resonant perturbation fields measurement in standing wave cavities, in proceedings of PAC 2009, Vancouver, Canada (2009).

[12] D. Alesini, Power coupling, Cern Accelerator School, Ebeltoft, Denmark, June 2010. 